\title{
Термоэлектрические свойства полуметаллических и полупроводниковых фольг и нитей $\mathrm{Bi}_{1-x} \mathrm{Sb}_{x}$
}

\author{
(C) А. Николаева ${ }^{1,2}$, Л. Конопко ${ }^{1,2}$, И. Гергишан ${ }^{1}$, К. Рогацкий $^{2}$, П. Стачовик ${ }^{2}$, А. Ежовски ${ }^{2}$, \\ В. Шепелевич ${ }^{3}$, В. Прокошин ${ }^{3}$, С. Гусакова ${ }^{3}$ \\ ${ }^{1}$ Инстутут электронной инженерии и нанотехнологии им. Д. Гицу, \\ МД-2028 Кишинев, Молдова \\ ${ }^{2}$ Институт низких температур и структурных исследований Польской академии наук, \\ 53-217 Вроцлав, Польша \\ ${ }^{3}$ Белорусский государственный университет, \\ 220030 Минск, Республика Беларусь \\ E-mail: A.Nikolaeva@nano.asm.md
}

(Поступила в Редакцию 20 декабря 2018 г.

В окончательной редакции 24 декабря 2018 г.

Принята к публикации 28 декабря 2018 г.)

\begin{abstract}
Представлены результаты экспериментальных исследований термоэлектрических свойств - проводимости, термоэдс, теплопроводности - микротекстурированных фольг и монокристаллических нитей на базе полуметаллических и полупроводниковых сплавов $\mathrm{Bi}_{1-x} \mathrm{Sb}_{x}$, в интервале температур 4.2-300 K. Установлено, что в нитях $\mathrm{Bi}-17 \mathrm{aT} \% \mathrm{Sb}$ энергетическая щель $\Delta E$ возрастает с уменьшением диаметра нитей $d$, что является проявлением квантового размерного эффекта. В области низких температур $(T<50 \mathrm{~K})$ в нитях c $d<0.4$ мкм проводимость возрастает вследствие существенного вклада высокопроводящих поверхностных состояний, характерных для топологических изоляторов. Впервые обнаружено, что теплопроводность полуметаллических фольг $\mathrm{Bi}-3 \mathrm{aT} \% \mathrm{Sb}$ в области низких температур на 2 порядка, а в полупроводниковых $\mathrm{Bi}-16 \mathrm{ar} \% \mathrm{Sb}$ на 1 порядок меньше, чем в массивных монокристаллах соответствующего состава, вследствие значительного рассеяния фононов на границах зерен и поверхности. Это привело к значительному возрастанию термоэлектрической эффективности и может быть использовано в миниатюрных термоэлектрических преобразователях энергии.
\end{abstract}

DOI: 10.21883/FTP.2019.05.47559.17

\section{1. Введение}

Сплавы $\mathrm{Bi}_{1-x} \mathrm{Sb}_{x}$ являются группой лучших низкотемпературных термоэлектрических и магнитотермоэлектрических материалов, в частности для охладителей и милливольтовой электроники [1]. Имеется ограниченный набор материалов, имеющих необходимые термоэлектрические, механические и другие свойства, необходимые для практических приложений. В настоящее время повышение термоэлектрической эффективности (ТЭЭ) $Z=\alpha^{2} \sigma / \chi$ связывается с новыми явлениями, такими как квантовый размерный эффект и недавно открытое новое состояние материи, известное как топологический изолятор (ТИ). В последнее время повышенный интерес к сплавам на базе $\mathrm{Bi}_{1-x} \mathrm{Sb}_{x}$ вызван предсказанным повышением термоэлектрической эффективности за счет квантового размерного эффекта в размерно-ограниченных структурах [2], что стимулировало большое количество теоретических и экспериментальных работ [3-5] в этом направлении. Кроме того, сплавы $\mathrm{Bi}_{1-x} \mathrm{Sb}_{x}$ в полупроводниковой области концентраций с инвертированным спектром [6] являются ТИ [7-9], в которых предсказывалось повышение ТЭЭ, когда химический потенциал расположен в области щели поверхностной зоны [10,11]. Таким образом, исследование термоэлектрических свойств в низкоразмерных системах сосредоточено на объектах 2 типов - моно- кристаллические размерно-ограниченные структуры, в которых реализуется эффект размерного квантования, и объемные микро- и нанокомпозиты. Возникновение большого числа границ раздела, которые эффективно рассеивают фононы, должно привести к существенному уменьшению теплопроводности, что приведет к существенному возрастанию ТЭЭ.

В данной работе представлены результаты экспериментальных исследований термоэлектрических свойств микротекстурированных фольг в полуметаллической и полупроводниковых фазах, а также монокристаллических нитей ТИ на базе полупроводниковых сплавов $\mathrm{Bi}-17 \mathrm{aT} \% \mathrm{Sb}$, в которых проявляются свойства ТИ и реализуется эффект размерного квантования.

\section{2. Образцы и методика эксперимента}

Фольги полуметаллических (Bi-3 aт\% $\mathrm{Sb})$ и полупроводниковых (Bi-9 aт\% $\mathrm{Sb}, \mathrm{Bi}-16 \mathrm{aT} \% \mathrm{Sb})$ сплавов изготавливались методом высокоскоростной кристаллизации капли расплава соответствующего состава на внутренней полированной поверхности вращающегося медного цилиндра, со скоростью кристаллизации $\sim 5 \cdot 10^{5} \mathrm{M} / \mathrm{c}[12]$, что обеспечивало равномерное распределение компонентов сплавов в объеме. Толщина фольг составляла $t=15-40$ мкм, рентгеноструктурные иссле- 
дования указывают на образование текстуры (1012) со средним размером зерна $\sim 9$ мкм. Зерна располагаются плоскостями параллельно поверхности фольги, а ось симметрии $C_{3}$ совпадает с нормалью к поверхности фольги. Монокристаллические нити $\mathrm{Bi}-17 \mathrm{aT} \% \mathrm{Sb}$ в стеклянной изоляции с диаметрами от 200 нм до 1000 мкм изготавливались методом литья из жидкой фазы по методу Улитовского [13,14]. Определяемая с помощью рентгеновской дифракции ориентация нитей указывает на направление $10 \overline{1} 1$ вдоль оси нити. При этом биссекторная ось $C_{1}$ отклонена на угол $19.5^{\circ}$ от оси нити в биссекторно-тригональной плоскости. Диаметр нитей определялся с помощью сканирующего электронного микроскопа.

Измерения гальвано- и термомагнитных свойств нитей и термоэдс фольг проводились двухконтактным методом с использованием InGa-эвтектики, хорошо смачивающей торцы, обеспечивая омические контакты. Измерение сопротивления и магнитосопротивления фольг проводилось четырехконтактным способом.

Для определения зависимости коэффициента теплопроводности от температуры исследованной фольги использовался метод теплового потока, основанный на законе Фурье:

$$
\mathbf{h}=-\chi \operatorname{grad} T,
$$

где $h$ - поток тепловой энергии через сечение образца, перпендикулярное потоку, $\chi-$ коэффициент теплопроводности.

Измерения теплопроводности проводились в Институте низких температур и структурных исследований ПАН, г. Вроцлав, Польша.

Температурная зависимость коэффициента теплопроводности фольги определена в диапазоне $5-300 \mathrm{~K}$. Полная ошибка измерений не превышала $6 \%$.

\section{3. Результаты и обсуждение}

Известно, что диаграммы вращения поперечного магнитосопротивления позволяют подтвердить монокристалличность образцов и установить направление ориентации кристаллографических осей.

На рис. 1 представлены диаграммы вращения по углу $\theta$ поперечного магнитосопротивления $\Delta R / R=R_{H}$ $-R_{0}(\theta)(\mathbf{H} \perp \mathbf{I})$ и магнитотермоэдс $\alpha(\theta),(\mathbf{H} \perp \operatorname{grad} \Delta T)$ фольг и нитей $\mathrm{Bi}_{1-x} \mathrm{Sb}_{x}$ при $80 \mathrm{~K}, H=0.4$ Тл.

Как видно из рис. $1, a, b$, магнитосопротивление монокристаллических нитей $\mathrm{Bi}-17 \mathrm{aT} \% \mathrm{Sb}$ имеет большую анизотропию магнитосопротивления $(60 \%)$ и анизотропию термоэдс $\Delta \alpha=20 \mathrm{M \kappa B} / \mathrm{K}$ при $H=0.4$ Тл (рис. 1, кривая 3), что подтверждает монокристалличность исследуемых нитей. Некоторая асимметрия (рис. 1, кривая 3) и наличие экстремумов при углах $\theta=90^{\circ}$ и $275^{\circ}$ указывают на отклонение оси нити от биссекторной оси [15].
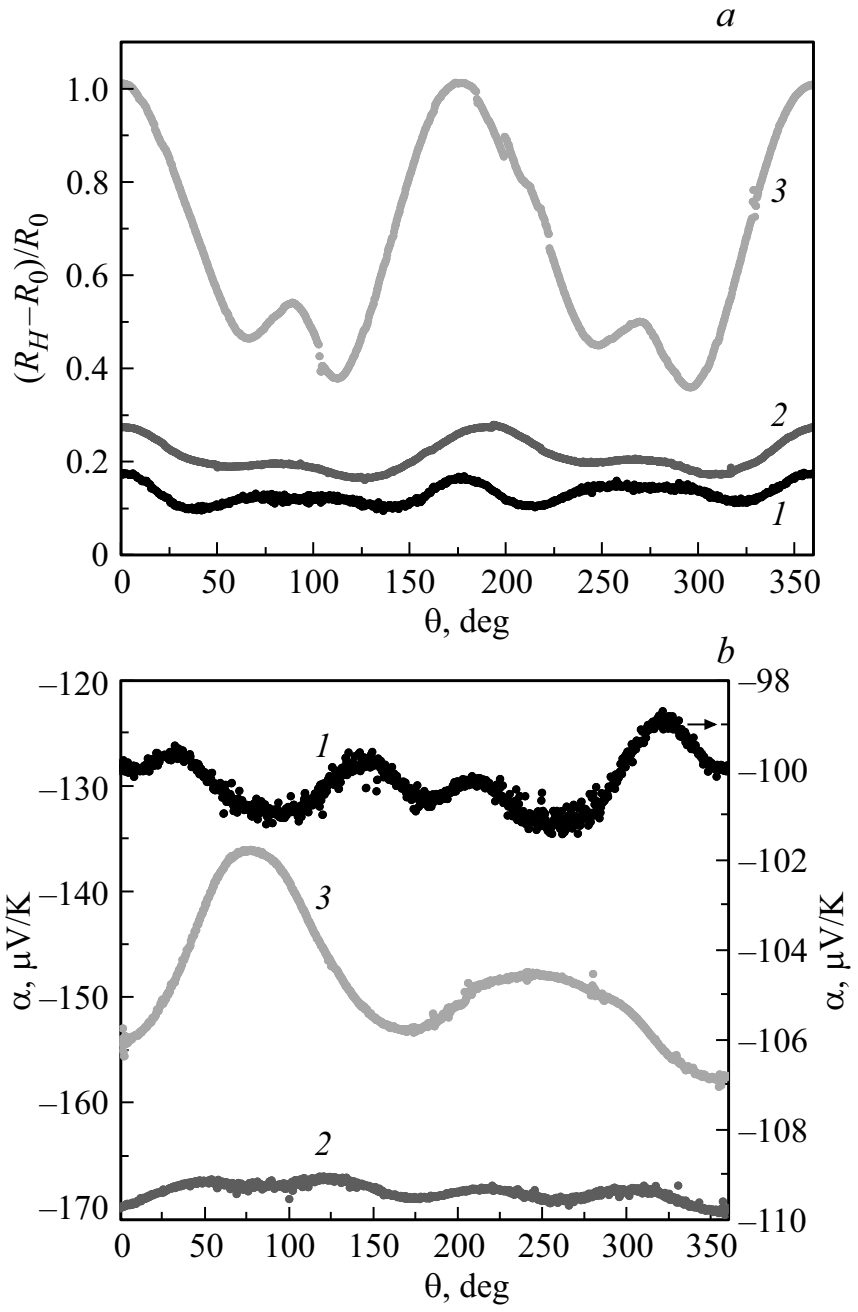

Рис. 1. Угловые диаграммы вращения поперечного магнитосопротивления $(a)$ и магнитотермоэдс $(b)$ фольг и нитей: $1-$ фольга $\mathrm{Bi}-3 \mathrm{aT} \% \mathrm{Sb}, t=12$ мкм; $2-$ фольга $\mathrm{Bi}-16$ ат\% $\mathrm{Sb}, t=23$ мкм; $3-$ нить $\mathrm{Bi}-17 \mathrm{aт} \% \mathrm{Sb}, d=2.1$ мкм при $\theta=0, \mathbf{H} \| C_{3}, H=0.4$ Тл, $T=80 \mathrm{~K}$.

Отсутствие анизотропии как магнитосопротивления, так и термоэдс $\alpha(\theta)$ в фольгах (рис. 1, кривые 1,2) подтверждает их микротекстурированную структуру.

В работе проведен комплекс исследований температурных зависимостей сопротивления, $R(T)$, термоэдс, $\alpha(T)$, теплопроводности, $\chi(T)$, в фольгах и нитях в интервале температур $4.2-300 \mathrm{~K}$ и рассчитаны температурные зависимости фактора мощности $\alpha^{2} \sigma(T)$ и термоэлектрической эффективности $Z T(T)$ для фольг полуметаллических и полупроводниковых сплавов $\mathrm{Bi}_{1-x} \mathrm{Sb}_{x}$.

На рис. 2,3 приведены температурные зависимости удельного сопротивления $\rho(T)$ и термоэдс $\alpha(T)$ фольг составов $\mathrm{Bi}-3 \mathrm{aT} \% \mathrm{Sb}, \mathrm{Bi}-9 \mathrm{aT} \% \mathrm{Sb}, \mathrm{Bi}-16 \mathrm{aT} \% \mathrm{Sb}$, а также монокристаллических нитей $\mathrm{Bi}-17 \mathrm{aT} \% \mathrm{Sb}$ в стеклянной оболочке с диаметрами $d=200,900$ нм. При $300 \mathrm{~K}$ удельное сопротивление возрастает от значения $1.5 \cdot 10^{-4}$ Ом · см для полуметаллического состава фольг $\mathrm{Bi}-3 \mathrm{aT} \% \mathrm{Sb}$ до значения $\sim 1.9 \cdot 10^{-4} \mathrm{OM} \cdot$ см для полу- 


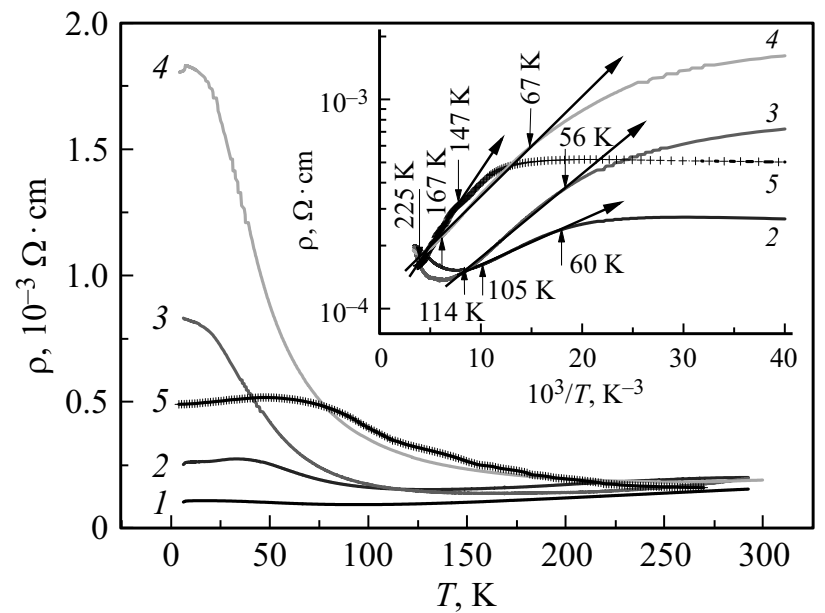

Рис. 2. Температурные зависимости удельного сопротивления $\rho(T)$ фольг и нитей. Фольги: $1-\mathrm{Bi}-3 \mathrm{aT} \% \mathrm{Sb}, t=12$ мкм; $2-\mathrm{Bi}-9$ ат $\% \mathrm{Sb}, t=27$ мкм; $3-\mathrm{Bi}-16$ ат\% $\mathrm{Sb}, t=23$ мкм. Нити: $4-\mathrm{Bi}-17 \mathrm{aT} \% \mathrm{Sb}, d=900 \mathrm{нм}, 5-\mathrm{Bi}-17 \mathrm{aT} \% \mathrm{Sb}$, $d=200$ нм. На вставке - зависимость $\rho\left(10^{3} / T\right)$ в логарифмическом масштабе.

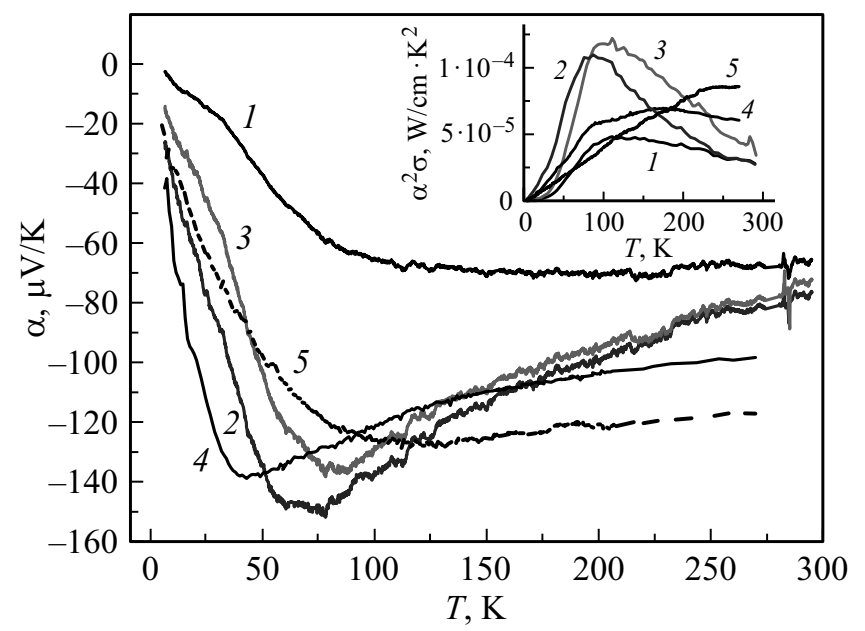

Рис. 3. Температурные зависимости термоэдс $\alpha(T)$ фольг и нитей. Фольги: $1-\mathrm{Bi}-3 \mathrm{aT} \% \mathrm{Sb}, t=12$ мкм; $2-\mathrm{Bi}-9 \mathrm{aT} \% \mathrm{Sb}$, $t=27$ мкм; $3-\mathrm{Bi}-16 \mathrm{aT} \% \mathrm{Sb}, t=23$ мкм. Нити: $4-$ $\mathrm{Bi}-17 \mathrm{aT} \% \mathrm{Sb}, d=900 \mathrm{Hм}, 5-\mathrm{Bi}-17 \mathrm{aT} \% \mathrm{Sb}, d=200 \mathrm{нм}$. На вставке - температурная зависимость силового фактора $\alpha^{2} / \sigma(T)$.

проводниковых сплавов фольг и нитей и практически не зависит от диаметра нитей $d$.

C уменьшением температуры сопротивление возрастает и на зависимостях $\rho(T)$ имеются экспоненциальные участки $\rho(T) \propto \exp \left(\Delta E / k_{\mathrm{B}} T\right)$ (см. вставку на рис. 2). Наклон экспоненциальных участков зависит от состава сплавов фольг и от диаметpa нитей $\mathrm{Bi}-17 \mathrm{aT} \% \mathrm{Sb}$. Из линейных зависимостей $\ln \rho\left(10^{3} / T\right)$ была определена термическая щель, которая для фольг $\mathrm{Bi}-9 \mathrm{aT} \% \mathrm{Sb}$ и $\mathrm{Bi}-16 \mathrm{aT} \% \mathrm{Sb}$ составляла $\Delta E=(8 \pm 1)$ мэВ и $(16 \pm 1)$ мэВ соответственно. Для монокристаллических нитей $\mathrm{Bi}-17 \mathrm{aT} \% \mathrm{Sb}$ c $d=900$ нм ширина щели $\Delta E=(19 \pm 1)$ мэВ и находится в хорошем согласии со значениями для массивных образцов аналогичного состава. Для нитей с $d=200$ нм значение ширины запрещенной зоны в сравнении с нитями с $d=900$ нм возрастает, до $\Delta E=(36 \pm 1)$ мэВ. Такая зависимость $\Delta E$ от диаметра нитей $d$ связана с проявлением квантового размерного эффекта, предсказанного в работах $[4,16]$ и наблюдаемого в нитях чистого Ві и сплавах $\mathrm{Bi}-3 \mathrm{aT} \% \mathrm{Sb}[13,14]$. Следует обратить внимание на то, что на температурных зависимостях $\rho(T)$ нитей $\mathrm{Bi}-17 \mathrm{aT} \% \mathrm{Sb}$ c $d=200$ нм в области температур $T<70 \mathrm{~K}$ наблюдается насыщение роста сопротивления $\rho(T)$ и имеет место тенденция к уменьшению сопротивления (кривая 5 ) при $T \rightarrow 4.2 \mathrm{~K}$.

Как было убедительно показано в работе [17], такая зависимость $\rho(T)$ в полупроводниковых нитях связана с проявлением свойств топологического изолятора.

На рис. 3 представлены температурные зависимости термоэс исследуемых образцов в интервале температур 4.2-300 K. Термоэдс отрицательна во всем интервале температур и, как в массивных образцах, максимальные значения термоэдс достигаются в фольгах и нитях полупроводниковых сплавов $\mathrm{Bi}-17 \mathrm{aT} \% \mathrm{Sb}$ и $\mathrm{Bi}-9 \mathrm{aT} \% \mathrm{Sb}$ в области температур 50-100 K. С уменьшением диаметра нитей $d$ величина максимального значения термоэдс уменьшается и положение максимума термоэдс смещается в область более высоких температур. Уменьшение термоэдс в тонких нитях полупроводниковых сплавов $\mathrm{Bi}-17 \mathrm{aT} \% \mathrm{Sb}$ связано с влиянием поверхностного рассеяния на длину свободного пробега электронов, приводящим в области низких температур к уменьшению электронного вклада в термоэдс.

Рассчитанные значения фактора мощности $\alpha^{2} \sigma(T)$ по экспериментальным данным $\alpha(T)$ и $\sigma(T)$ приведены на вставке к рис. 3. Максимальные значения $1.2 \cdot 10^{-4} \mathrm{BT} / \mathrm{cm} \cdot \mathrm{K}^{2}$ достигаются в фольгах $\mathrm{Bi}-9 \mathrm{aT} \% \mathrm{Sb}$ и $\mathrm{Bi}-16 \mathrm{aT} \% \mathrm{Sb}$, а также в нитях $\mathrm{Bi}-17 \mathrm{aT} \% \mathrm{Sb}$, $d=900$ нм, в области температур 70-120 К (см. вставку на рис. 3), что превосходит значения, полученные на массивных образцах соответствующего состава. В области высоких температур, $T>200 \mathrm{~K}$, максимальное значение фактора мощности имеет место в самых тонких нитях $\mathrm{Bi}-17$ ат\% $\%$ с с $d=200$ нм.

Экспериментальные зависимости теплопроводности $\chi(T)$ в интервале температур $4.2-300 \mathrm{~K}$ для фольг составов $\mathrm{Bi}-3 \mathrm{aT} \% \mathrm{Sb}$ и $\mathrm{Bi}-16 \mathrm{aT} \% \mathrm{Sb}$ приведены на рис. 4 (кривые 1,2). Для сравнения приведены также $\chi(T)$ массивных образцов из работы [18].

В области температур $200-300 \mathrm{~K}$ значение теплопроводности фольг практически совпадает со значениями, полученными на массивных образцах. В массивных образцах при температурах $T<200 \mathrm{~K}$ характер кривых $\chi(T)$ существенно изменяется - происходит резкое возрастание на 2 порядка теплопроводности полуметаллических образцов $\mathrm{Bi}-3 \mathrm{ar} \% \mathrm{Sb}$ и на порядок 


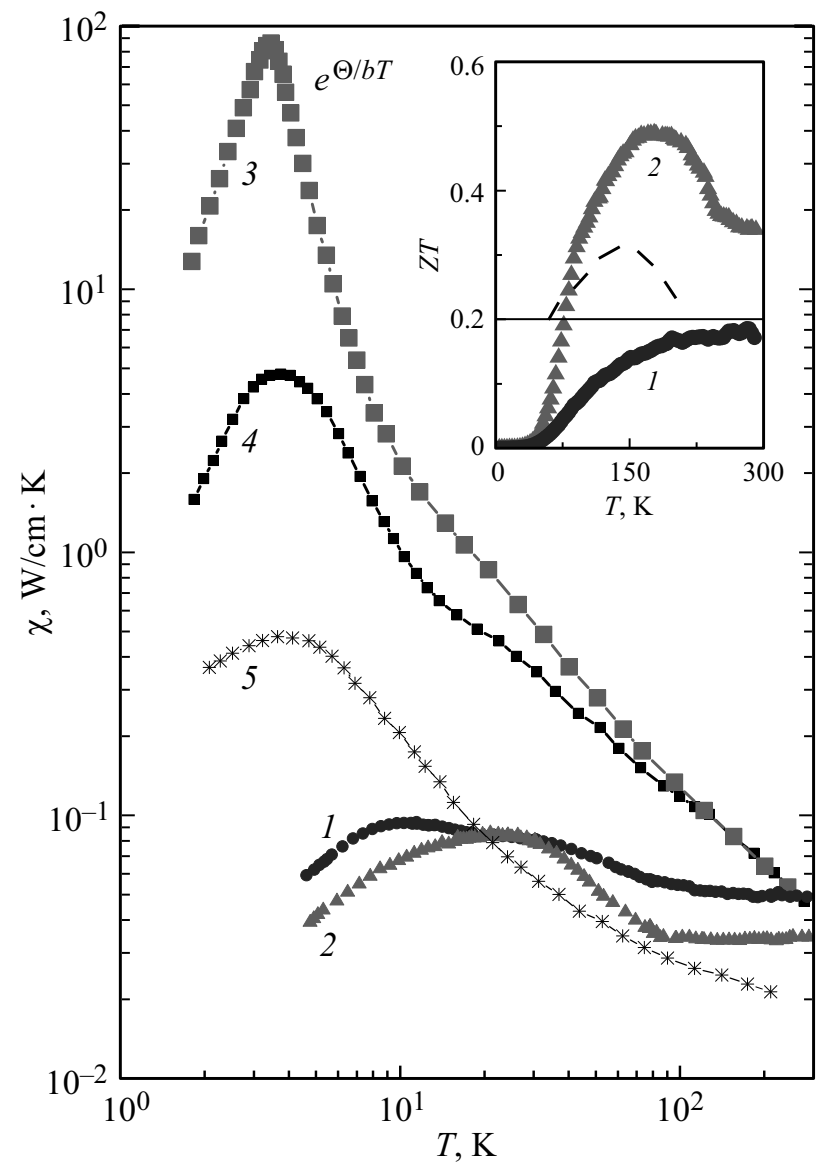

Рис. 4. Температурные зависимости теплопроводности $\chi(T)$ фольг: $1-\mathrm{Bi}-3 \mathrm{aT} \% \mathrm{Sb}, t=12$ мкм; $2-\mathrm{Bi}-16 \mathrm{aT} \% \mathrm{Sb}$, $t=23$ мкм; 3, 4,5 - экспериментальные кривые массивных образцов: $3-\mathrm{Bi}, 4-\mathrm{Bi}-1 \mathrm{aT} \% \mathrm{Sb}, 5-\mathrm{Bi}-12 \mathrm{aT} \% \mathrm{Sb}$ [18]. На вставке - температурные зависимости термоэлектрической эффективности $Z T(T)$. Штриховая кривая соответствует массивному образцу $\mathrm{Bi}-12 \mathrm{aT} \% \mathrm{Sb}$ [18].

полупроводниковых $\mathrm{Bi}-12 \mathrm{aT} \% \mathrm{Sb}$ (рис. 4, кривые 4,5 соответственно).

В исследуемых фольгах аналогичного состава резкий рост теплопроводности (при $T<100 \mathrm{~K}$ ) подавляется и так называемый ,диэлектрический максимум““, проявляющийся у массивных образцов при температурах $3-4 \mathrm{~K}$, смещается в область температур $10-30 \mathrm{~K}$ (рис. 4, кривые 1,2 ). Уменьшение теплопроводности на 2 порядка по сравнению с массивными образцами $\mathrm{Bi}-\mathrm{Sb}$ наблюдается при $T<200 \mathrm{~K}$ для полуметаллических фольг $\mathrm{Bi}-3 \mathrm{aT} \% \mathrm{Sb}$ и на один порядок для полупроводниковых фольг $\mathrm{Bi}-16 \mathrm{aT} \% \mathrm{Sb}$.

Известно, что на температурной зависимости $\chi(T)$ массивных образцов чистого Ві наблюдается максимум теплопроводности при $T_{\max }=3.5 \mathrm{~K}$. При температурах выше температуры максимума из-за наличия процессов переброса ( $U$-процессы) наблюдается экспоненциальная зависимость теплопроводности Ві от температуры [19]. Экспоненциальный рост теплопроводности с пониже- нием температуры $<100 \mathrm{~K}$ обусловлен экспоненциальным ростом средней длины свободного пробега фононов $\left(l \propto e^{\Theta / b T}\right)$ (рис. 4, кривая 3$)$, где $\Theta=120 \mathrm{~K}-$ температура Дебая. При температурах $T<15 \mathrm{~K}$ длина свободного пробега фононов в висмуте растет экспоненциально только до тех пор, пока не станет сравнимой с величиной поперечного размера образца [20]. В результате при дальнейшем понижении температуры из-за рассеяния фононов на границах образца в температурной зависимости теплопроводности появляется максимум.

В фольгах, имеющих зернистую структуру с диаметром зерен $\sim 9$ мкм и с наличием двойников, реализуется среда с большим числом границ раздела, что приводит к дополнительному рассеянию фононов на границах зерен и соответственно к значительному уменьшению теплопроводности.

Результаты исследования температурных зависимостей удельного сопротивления $\rho(T)$, термоэдс $\alpha(T)$ и теплопроводности $\chi(T)$ позволили рассчитать температурные зависимости термоэлектрической эффективности $Z T(T)=\alpha^{2} \sigma / \chi(T)$ (см. вставку на рис. 4).

Как видно из рис. 4 , максимальные значения термоэлектрической эффективности $Z T$ наблюдаются в фольгах полупроводниковых сплавов $\mathrm{Bi}-16 \mathrm{aT} \% \mathrm{Sb} n$-типа проводимости в интервале температур $120-220 \mathrm{~K}$, $Z T=0.5$, что почти в 2 раза превышает максимальные значения, полученные на полупроводниковых $n$-сплавах $\mathrm{Bi}-12 \mathrm{aT} \% \mathrm{Sb}$ и в пленках аналогичных составов и аналогичных кристаллографических ориентаций [21-23].

Таким образом, исследуемые фольги полупроводникового сплава $\mathrm{Bi}-16 \mathrm{aT} \% \mathrm{Sb}$, имеющие термоэлектрическую добротность $Z T \approx 0.5$ в области $80<T<150 \mathrm{~K}$, могут использоваться в качестве $n$-ветвей низкотемпературных миниатюрных термоэлектрических преобразователей энергии.

\section{4. Заключение}

Проведено комплексное исследование температурных зависимостей термоэдс $\alpha(T)$, удельного сопротивления $\rho(T)$ и теплопроводности $\chi(T)$ микротекстурированных фольг и монокристаллических нитей на базе полуметаллических и полупроводниковых сплавов $\mathrm{Bi}_{1-x} \mathrm{Sb}_{x}$. Обнаружено, что теплопроводность в полуметаллических фольгах $\mathrm{Bi}-3 \mathrm{ar} \% \mathrm{Sb}$ в области низких температур на 2 порядка, а в полупроводниковых $\mathrm{Bi}-16 \mathrm{aT} \% \mathrm{Sb}$ на один порядок меньше, чем в массивных образцах аналогичного состава. Это связано с возрастанием рассеяния фононов на поверхности и на границах зерен и приводит к росту термоэлектрической эффективности в области температур 120-200 K.

Установлено, что энергетическая щель в тонких полупроводниковых нитях $\mathrm{Bi}-17 \mathrm{aT} \% \mathrm{Sb}(d=200$ нм $)$ возрастает благодаря проявлению квантового размерного эффекта, а в области низких температур обнаружено уменьшение удельного сопротивления, что связано с 
проявлением свойств ТИ. Показано, что уменьшение диаметра нитей $d$ полупроводниковых сплавов $\mathrm{Bi}_{1-x} \mathrm{Sb}_{x}$ приводит к росту фактора мощности при $T>200 \mathrm{~K}$.

Работа поддержана Институционным проектом IIEN 15.817.02.09A.

\section{Список литературы}

[1] P. Jandl, U. Birkholz. J. Appl. Phys., 76 (11), 7351 (1994).

[2] L.D. Hicks, M.S. Dresselhaus. Phys. Rev. B, 47, 16631 (1993).

[3] O. Rabin, Y.-M. Lin, M.S. Dresselhaus. Appl. Phys. Lett., 79 (1), 81 (2001).

[4] Sh. Tang, M.S. Dresselhaus. Phys. Rev. B, 89, 045424 (2014).

[5] R. Venkatasubramanian, E. Siivola, T. Colpitts, B. O'Quinn. Nature, 413 (6856), 597 (2001).

[6] Г.А. Миронова, М.В. Судакова, Я.Г. Пономарев. ЖЭТФ, 78 (5), 1832 (1980).

[7] Fu Liang, C.L. Kane, E.J. Mele. Phys. Rev. Lett., 98, 106803 (2007).

[8] A.A. Taskin, Kouji Segawa, Yoichi Ando. Phys. Rev. B, 82, 121302(R) (2010).

[9] R. Takahashi, S. Murakami. Semicond. Sci. Technol., 27 (12), 124500 (2012).

[10] D. Hsieh, D. Qian, L. Wray, Y. Xia, Y.S. Hor, R.J. Cava, M.Z. Hasan. Nature, 452 (7190), 970 (2008).

[11] Dong-Xia Qu, K. Roberts Sarah, George F. Chapline. Phys. Rev. Lett., 111, 176801 (2013).

[12] A.V. Demidchik, V.G. Shepelevich. Inorg. Mater., 40 (4), 391 (2004).

[13] A. Nikolaeva, T.E. Huber, D. Gitsu, L. Konopko. Phys. Rev. B, 77, 035422 (2008).

[14] A.A. Nikolaeva, L.A. Konopko, T.E. Huber, P.P. Bodiul, I.A. Popov. J. Sol. State Chem., 193, 71 (2012).

[15] И.М. Пилат, С.В. Чайка, Н.В. Кругова, С.И. Пироженко. ФTT, 17 (1), 176 (1975).

[16] J.P. Heremans. Acta Phys. Polon. A, 108 (4), 609 (2005).

[17] L.A. Konopko, A.A. Nikolaeva, T.E. Huber, J.-P. Ansermet. J. Low Temp. Phys., 185 (5), 673 (2016).

[18] В.Д. Каган, Н.А. Редько. ФТТ, 11, 3480 (1992).

[19] В.Н. Копылов, Л.П. Межов-Деглин. ЖЭТФ, 65 (8), 720 (1973).

[20] П.П. Бодюл, М.П. Бойко, Н.А. Редько. ФТТ, 28 (10), 3182 (1986).

[21] B. Lenoir, A. Dauscher, M. Cassat, Yu.I. Ravich, H. Sherrer. J. Phys. Chem. Sol., 59, 129 (1998).

[22] Н.А. Родионов, Г.А. Иванов, Н.А. Редько. ФТТ, 24 (6), 1881 (1982).

[23] Shuang Tang, Mildred Dresselhaus. Nano Lett., 12 (4), 2021 (2012).

Редактор Л.В. Шаронова

\section{Thermoelectric properties semimetal and} semiconductor $\mathbf{B i}_{1-x} \mathbf{S b}_{x}$ foils and wires

\author{
A. Nikolaeva ${ }^{\mathbf{1}, 2}$, L. Konopko ${ }^{\mathbf{1 , 2}}$, I. Gherghishan ${ }^{\mathbf{1}}$, \\ K. Rogacki ${ }^{2}$, P. Stachowiak ${ }^{2}$, A. Jezowski ${ }^{2}$, \\ V. Shepelevich ${ }^{3}$, V. Prokoshin ${ }^{3}$, S. Gusakova ${ }^{3}$ \\ ${ }^{1}$ Ghitu Institute of Electronic Engineering \\ and Nanotechnologies, \\ MD-2028 Chisinau, Republica Moldova \\ ${ }^{2}$ Institute of Low Temperature and Structure Research, \\ Academy of Sciences, \\ 53-217 Wrocław, Poland \\ ${ }^{3}$ Belarusian State University, \\ 220030 Minsk, Republic of Belarus
}

\begin{abstract}
This paper reports the results of experimental studies of the thermoelectric properties (electrical conductivity, thermopower, and thermoconductivity) of microstructured foils and glass-insulated single-crystal wires based on semimetal and semiconductor $\mathrm{Bi}_{1-x} \mathrm{Sb}_{x}$ alloys in the temperature range of $4.2-300 \mathrm{~K}$. It has been found that in $\mathrm{Bi}-17 \mathrm{at} \% \mathrm{Sb}$ wires energy gap $\Delta E$ increases with decreasing wire diameter $d$; this finding is attributed to occurrence of the quantum size effect. At low temperatures $(T<50 \mathrm{~K})$, in the wires with $d<400 \mathrm{~nm}$ the electrical conductivity increases owing to a significant contribution of highly conductive surface states characteristic of topological insulators.

It has been first found that at low temperatures the thermal conductivity of the semimetal $\mathrm{Bi}-3 \mathrm{at} \% \mathrm{Sb}$ foils and the semiconductor $\mathrm{Bi}-16$ at $\% \mathrm{Sb}$ foils is lower than that in the bulk samples of the respective composition: by 2 orders of magnitude for the former and 1 order of magnitude for the latter. This finding is attributed to significant phonon scattering at grain boundaries and surfaces. This effect leads to a considerable enhancement of the thermoelectric figure of merit $Z T$ and can be used in miniature low-temperature thermoelectric energy converters.
\end{abstract}

